\title{
AREA AND HAUSDORFF DIMENSION OF JULIA SETS OF ENTIRE FUNCTIONS
}

\author{
CURT MCMULLEN
}

\begin{abstract}
We show the Julia set of $\lambda \sin (z)$ has positive area and the action of $\lambda \sin (z)$ on its Julia set is not ergodic; the Julia set of $\lambda \exp (z)$ has Hausdorff dimension two but in the presence of an attracting periodic cycle its area is zero.
\end{abstract}

1. Introduction. There is no known rational function $R$ whose Julia set $J$ has positive area and yet is not the whole Riemann sphere. (Indeed there is as yet no example for which $J$ is proved to be a proper subset of the sphere with Hausdorff dimension two.) On the other hand, when the forward orbits of the critical points do not accumulate on $J$, the map $R$ is expanding and Sullivan shows $\operatorname{dim}(J)<2$ (in fact $J$ has positive finite measure in its dimension $[\mathbf{S}]$ ). So disregarding the case $J=\hat{C}$, it is fairly hard to find large Julia sets.

The purpose of this note is to describe a contrasting situation for some entire functions. We consider two families of functions:

$$
\begin{array}{ll}
\left\{f(z)=\lambda e^{z}: \lambda \neq 0\right\} & \text { (the "exponential family"), } \\
\{f(z)=\sin (\alpha z+\beta): \alpha \neq 0\} & \text { (the "sine family"). }
\end{array}
$$

Each of these families is "topologically complete", in the sense that any entire function topologically conjugate to a member of the family is already (conformally conjugate to) a member. The sine family represents the same conformal conjugacy classes as

$$
\left\{f(z)=\gamma e^{z}+\delta e^{-z}: \gamma \neq 0, \delta \neq 0\right\}
$$

so it contains functions conjugate to $\lambda \cos (z), \lambda \sinh (z)$, etc.

THEOREM 1.1. The Julia set of any member of the sine family always has positive area.

THEOREM 1.2. The Julia set of any member of the exponential family always has Hausdorff dimension two.

THEOREM 1.3. However, when $\lambda e^{z}$ has an attracting periodic cycle (e.g. $0<$ $\lambda<1 / e)$, the area of its Julia set is zero.

Examples of these Julia sets are rendered in Figures 1.1 and 1.2. The member of the exponential family shown has an attracting fixed point.

Since the Julia sets for members of the sine family have positive area, one can study their measurable dynamics.

Received by the editors March 28, 1986.

1980 Mathematics Subject Classification (1985 Revision). Primary 30D05; Secondary 58F13.

Research supported in part by NSF grant 8120790 . 


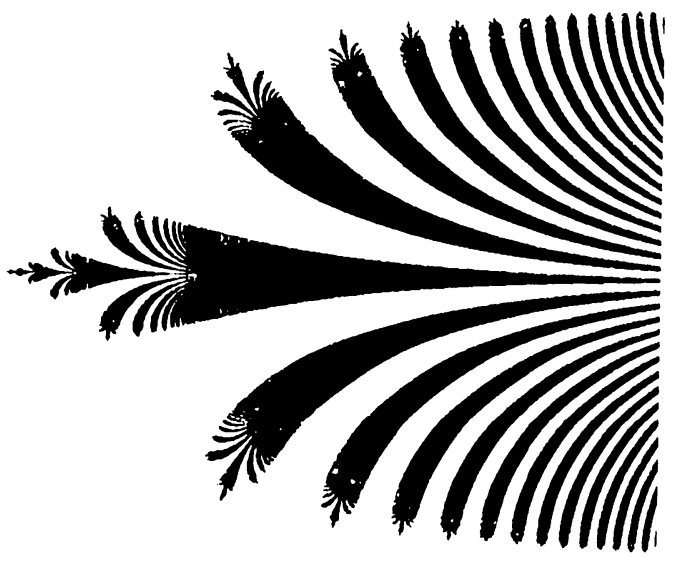

FIGURE 1.1. Julia set of $f(z)=0.3 \exp (z)$

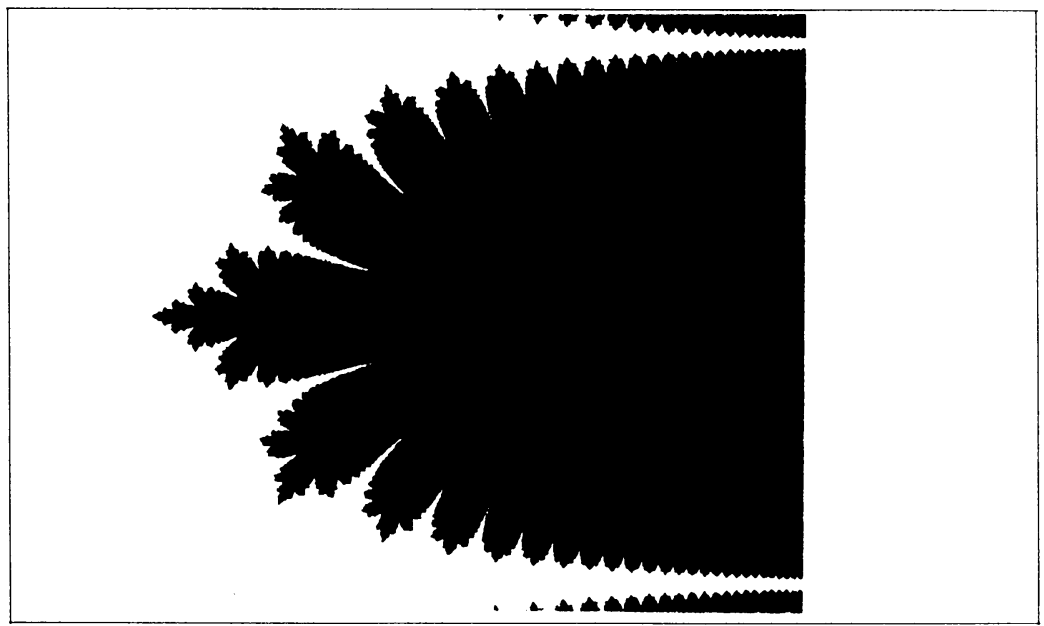

FIGURE 1.2. Julia set of $f(z)=0.7 \sinh (z)$

THEOREM 1.4. The action of $f(z)$ on its Julia set is not ergodic for any member of the sine family.

REMARKS. (1) The study of iterated entire functions begins with Fatou $[\mathbf{F}]$; some of the more recent developments, especially concerning the exponential family, appear in $\left[\mathbf{B 1}, \mathbf{B 2}, \mathbf{B R}, \mathbf{D K}\right.$, and GGS]. For $f(z)=e^{z}$, the Julia set is the whole plane $[\mathbf{M}]$ but the measurable dynamics are more complicated than for $\sin (\alpha z+\beta)$, due to the large contraction in the left half-plane. M. Ljubich has reportedly established the nonergodicity of $e^{z}$.

(2) In addition to the exponential and sine families, it is natural to consider the family

$$
\{f(z)=\cos (\sqrt{\alpha z+\beta}): \alpha \neq 0\} \quad \text { (the "cosine-root" family). }
$$

This family is also topologically complete. Taken together, these three families represent exactly those entire functions which arise as infinite sheeted coverings 
of noncompact orbifolds of Euler characteristic zero. (The exponential and sine families give universal coverings of the $(\infty, \infty)$ and $(\infty, 2,2)$ orbifolds respectively; the cosine family gives the covering of the $(\infty, 2,2)$ orbifold associated to an infinite cyclic subgroup of the infinite dihedral group.)

The arguments applied to $\sin (\alpha z+\beta)$ can be used to prove analogous results for the cosine-root family: every member of that family also has a Julia set of positive measure on which the action of $f$ is not ergodic. Since the proofs parallel those for $\sin (\alpha z+\beta)$ (but are slightly less transparent) we do not reproduce them here.

ADDED IN PROOF. A generalization of Theorem 3.1 has been announced by Eremenko and Ljubich [EL].

OUTLINE OF THE PAPER. We begin by developing simple criteria for subsets of $\mathbf{R}^{n}$ to have positive volume or Hausdorff dimension $n(\S 2)$, and by discussing the distortion lemma for expanding dynamical systems $(\S 3)$. These results are then applied to the Julia sets of $\lambda e^{z}$ and $\sin (\alpha z+\beta)$ to prove Theorems 1.1 and $1.2(\S \S 4$, $5)$. In $\S 6$ we discuss distortion properties of "expanding" entire functions (analogous to hyperbolic or Axiom A rational functions); it turns out that our hypothesis in Theorem 1.3 is exactly that $\lambda e^{z}$ is expanding. $\S 7$ completes the proof of Theorem 1.3 , and $\S 8$ discusses the measurable dynamics of $\sin (\alpha z+\beta)$. We have included an appendix which gives a growth criterion for points to be in the Julia set of an entire function of finite type. This criterion is used in many of the proofs.

ACKNOWLEDGMENTS. The results here are partly based on general arguments used by many authors, among them Douady, Hubbard, Ljubich and Sullivan. I am grateful to Bob Devaney for suggesting that the Julia set of $\lambda \sin (z)$ should have positive area.

I would like to thank the Math Sciences Research Institute for its hospitality while this work was being completed, and the Lawrence Berkeley Laboratories for the use of its computing facilities.

2. Volume and Hausdorff dimension. To study the Julia sets, we will construct explicit subsets of them by nested intersection of dynamically defined sets. In this section we develop general criteria which ensure that the resulting set has positive measure or dimension two.

For $k=1,2, \ldots$, let $E_{k}$ denote a finite collection of disjoint compact subsets $F$ of $\mathbf{R}^{n}$; and let $\bar{E}_{k}$ denote the compact set obtained as the union of the elements of $E_{k}$. We assume

(a) every $F \in E_{k+1}$ is contained in a (unique) $F^{\prime} \in E_{k}$; and

(b) every $F \in E_{k}$ contains at least one element of $E_{k+1}$.

Consider the set $E=\bigcap_{k=1}^{\infty} \bar{E}_{k}$. Assume that for all $k$ and all $F$ in $E_{k}$,

$$
\operatorname{density}\left(\bar{E}_{k+1}, F\right):=\frac{\operatorname{Vol}\left(\bar{E}_{k+1} \cap F\right)}{\operatorname{Vol}(F)} \geq \Delta_{k}
$$

and

$$
\operatorname{diameter}(F) \leq d_{k}
$$

Here Vol denotes $n$-dimensional volume. Then it is easy to establish

PROPOSITION 2.1.

$$
\operatorname{Density}\left(E, \bar{E}_{1}\right) \geq \prod_{k=1}^{\infty} \Delta_{k}
$$


In particular, if the product is greater than zero then $E$ has positive volume. On the other hand, if the product of the densities tends to zero, but much slower than $d_{k}$, then $E$ still has Hausdorff dimension $n$.

PROPOSITION 2.2.

$$
\varlimsup_{k \rightarrow \infty} \frac{\sum_{i=1}^{k+1}\left|\log \Delta_{i}\right|}{\left|\log d_{k}\right|} \geq n-\operatorname{dim}(E) .
$$

In particular, if the limit above is zero then the Hausdorff dimension of $E$ is equal to the ambient dimension $n$.

ProOF. For simplicity we rescale so that the $n$-dimensional volume of $\bar{E}_{1}$ is one. Construct a sequence of probability measures $\mu_{k}$ as follows: let $\mu_{1}$ be the restriction of $n$-dimensional volume measure to $\bar{E}_{1}$; and construct $\mu_{k+1}$ inductively so that within each $F \in E_{k}, \mu_{k+1}$ is proportional to volume measure on $\bar{E}_{k+1}$, scaled so that $\mu_{k+1}\left(\bar{E}_{k+1} \cap F\right)=\mu_{k}(F)$. These measures form a martingale relative to the collections $E_{k}$ in the sense that (by the above) $\mu_{m}(F)$ for $F$ in $E_{k}$ is the same for all $m \geq k$. Let $\mu$ denote a weak limit of the $\mu_{k}$ (in fact it is unique). Then $\mu$ is supported on $E$, and for $F \in E_{k}$,

$$
\mu(F) \leq \operatorname{Vol}(F) /\left(\Delta_{1} \Delta_{2} \cdots \Delta_{k}\right) .
$$

(The measure of $F$ is just its volume scaled up by the relative sparseness of $\bar{E}_{i+1}$ in $\bar{E}_{i}$, for $i=1,2, \ldots, k$.)

Consider a ball $B$ of radius $r$ in $\mathbf{R}^{n}$. To prove $E$ has dimension at least $\delta$, it suffices to show $\mu(B) \leq C(\delta) r^{\delta}$ (this is the easy half of Frostman's Lemma, see $[\mathbf{K S}])$.

Choose $k$ so that $d_{k}>r \geq d_{k+1}$, and let $\bar{B}$ denote the union of all sets $F \in E_{k+1}$ which meet $B$. Then the diameter of $\bar{B}$ is not more than twice that of $B$, so by the estimate above,

$$
\mu(B) \leq \mu(\bar{B}) \leq \frac{\operatorname{Vol}(\bar{B})}{\left(\Delta_{1} \Delta_{2} \cdots \Delta_{k+1}\right)} \leq C r^{\delta}\left[\frac{d_{k}^{n-\delta}}{\left(\Delta_{1} \Delta_{2} \cdots \Delta_{k+1}\right)}\right]
$$

where $C$ is a constant depending on $\delta$ and the volume of a unit sphere in dimension $n$. Whenever $n-\delta$ is greater than the $\varlimsup$ im in the statement of the proposition, the quantity in brackets tends to zero as $k \rightarrow \infty$, so in particular it is bounded above. Thus $\mu$ satisfies an estimate of the required form, so the Hausdorff dimension of $E$ is at least $\delta$, establishing the proposition.

3. Distortion in expanding dynamics: Telescopes. Let $D$ be a bounded open subset of the complex plane and let $f: D \rightarrow C$ be a map. We say $f$ has bounded distortion if for distinct $x$ and $y$ in $D$,

$$
c<|f(x)-f(y)| /|x-y|<C
$$

where $c$ and $C$ are positive constants depending only on $f$. The ratio $C / c=L(f)$ measures the amount of distortion of $f$; it is is near 1 , we say $f$ has small distortion. Small distortion implies $f$ nearly preserves relative lengths and areas. For example, if $X$ is a measurable subset of $D$, then

$$
\operatorname{density}(X, D)=\frac{\operatorname{Area}(X)}{\operatorname{Area}(D)} \leq L(f)^{2} \operatorname{density}(f(X), f(D)) .
$$


For a conformal map $f(z)$ a differential quantity related to distortion is the nonlinearity of $f$, measured by the rate of change of its derivative, i.e. by the quantity $\log \left(f^{\prime}\right)^{\prime}=f^{\prime \prime} / f^{\prime}$. If $D$ is a ball or a square and

$$
N(f)=\left(\sup _{z \in D}\left|f^{\prime \prime}(z) / f^{\prime}(z)\right|\right) \times(\operatorname{diameter}(D))
$$

is small, then $f$ has small distortion $[\mathbf{J}]$; in fact when $N(f)$ is near $0, L(f)$ is bounded by $1+O(N(f))$. (The exact condition needed here is that $D$ is a $K$-quasidisc, and the relation between nonlinearity and distortion depends only on the constant $K$ $[\mathbf{G}]$.)

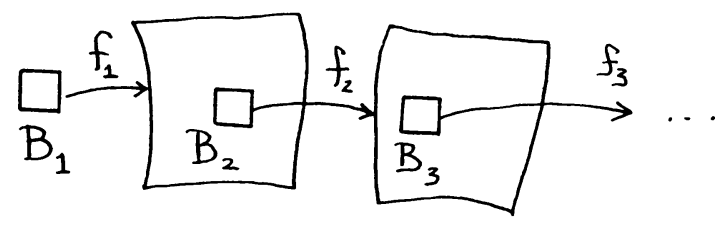

FIGURE 3.1. A telescope of expanding maps

A well-known principle in dynamics is that bounded nonlinearity combined with uniform expansion allows one to control distortion over any number of iterates. A form of this principle suited to our applications is the following: let $\left\langle B_{i}, i=\right.$ $1, \ldots, n\rangle$ be a sequence of squares of side $r$ in the plane, and $f_{i}$ a sequence of conformal maps such that $f_{i}\left(B_{i}\right)$ contains $B_{i+1}$. This chain of maps forms a sort of "telescope" (see Figure 3.1).

Assume that the maps $f_{i}$ have uniform expansion and bounded nonlinearity, that is, for all $i$,

$$
\left|f_{i}^{\prime}\right|>\alpha>1 \text { and }\left|f_{i}^{\prime \prime} / f_{i}^{\prime}\right|<M<\infty,
$$

and assume that $r$ (the size of the squares) is small enough to assure that each $f_{i}$ has uniformly bounded distortion on $B_{i}$. (Since $N\left(F_{i} \mid B_{i}\right) \leq M r$, this will hold whenever $r$ is sufficiently small.) Let $D=f_{n}\left(B_{n}\right)$ and let $F=\left(f_{n} \circ \cdots \circ f_{1}\right)^{-1}: D \rightarrow$ $B_{1}$ denote the inverse of the composition of all of the $f_{i}$.

Proposition 3.1 (The Distortion Lemma). The distortion of $F$ is bounded by a constant depending on $r, \alpha$ and $M$ (the size of the squares, the amount of expansion and the nonlinearity), but not on $n$.

PROOF. Uniform expansion insures the diameters of the successive preimages of $D$ under the $f_{i}$ go to zero at a geometric rate, so the same is true of $N()$ of the restriction of $f_{i}^{-1}$ to these sets. Using the relation $L(f)=1+O(N(f))$ and $L(f g) \leq L(f) L(g)$, we conclude that $L(F)$ is controlled by the sum of a geometric series which has a finite bound independent of $n$.

4. The Julia set of $\sin (\alpha z+\beta)$. For convenience, we will actually deal with $f(z)=\gamma e^{z}+\delta e^{-z}$, which is conjugate to $\sin (\alpha z+\beta)$ by an affine change of coordinates.

Consider the region

$$
R(h)=\{z:|\operatorname{Re}(z)|>h\}
$$


For $z$ in $R(h), h$ large, $\left|f^{\prime}\right|>O\left(e^{h}\right)$ and $\left|f^{\prime \prime} / f^{\prime}\right| \approx 1$. Whenever we consider a region $R(h)$, we will always take $h$ large enough that $f$ is expanding by a definite factor and the nonlinearity is near 1 .

In such an $R(h)$, we define a box to be a square region with sides of length $r$, where $r$ is a fixed constant chosen small enough to guarantee small distortion-in particular, that the image of the box under $f$ is again nearly square. (It is possible to choose such an $r$ by bounded nonlinearity.)

We now estimate the size of the set of points whose iterates tend to infinity at a given rate. Let $h_{k}$ be a sequence of positive numbers, a candidate for the growth of $\left|\operatorname{Re} f^{k}(z)\right|$.

Proposition 4.1. For $B_{0}$ a box in $R\left(h_{0}\right)$, the set of $z$ in $B_{0}$ such that $f^{k}(z) \in$ $R\left(h_{k}\right)$ for all $k$ has density at least

$$
\prod_{k=0}^{\infty}\left(1-O\left(h_{k+1} / e^{h_{k}}\right)\right) .
$$

Proof. Let $B$ be a box in $R\left(h_{k}\right)$. Then the set $f(B) \cap R\left(h_{k+1}\right)$ can itself be packed with disjoint boxes whose total density in $f(B)$ exceeds $1-O\left(h_{k+1} / e^{h_{k}}\right)$. This follows from the fact that $f(B) \cap R\left(h_{k+1}\right)$ looks very nearly like a square of side at least $O\left(e^{h}\right)$, possibly with a strip of width $2 h_{k+1}$ deleted. We will refer to the boxes in such a packing as $\operatorname{pack}(f(B))$.

We now define collections $E_{k}$ of subsets of $B_{0}$ which satisfy the nesting conditions of $\S 2$. Let $E_{0}=\left\{B_{0}\right\}$ and define inductively

$$
E_{k}=\left\{G: G \subset F \in E_{k-1}, \text { and } f^{k}(G) \in \operatorname{pack}\left(f^{k} F\right)\right\} .
$$

That is, $E_{k}$ consists of preimages of the boxes packing $f^{k}(F) \cap R\left(h_{k}\right)$ for each $F$ in $E_{k-1}$ (see Figure 4.1). Then $E=\bigcap E_{k}$ consists of points for which $f^{k}(z) \in R\left(h_{k}\right)$ for all $k$.

Since

$$
\text { Density }\left(\bigcup \operatorname{pack}\left(f^{k} F\right), f^{k} F\right) \geq 1-O\left(h_{k+1} / e^{h_{k}}\right),
$$

we conclude by the distortion lemma that the same is true (with a different constant in $O\left(\right.$ ) for density $\left(\bar{E}_{k+1}, F\right)$. Then Proposition 2.1 applies to complete the proof.

PROOF OF THEOREM 1.1 (The Julia set of $\sin (\alpha z+\beta)$ has positive area). By the above, if we choose $h_{k} \rightarrow \infty$ such that $\sum_{k=0}^{\infty} h_{k+1} / e^{h_{k}}$ is sufficiently small, we will have a set of positive density in $B$ such that for all $k, \operatorname{Re}(z)>h_{k}$. For example, if we take $h_{k}=2 g^{k}(x)$, where $g(x)=e^{x}$, the sum above becomes $\sum_{k=0}^{\infty} 2 / g^{k+1}(x)$ and choosing $x$ large, this sum becomes extremely small.

Thus there is always a set of positive area in $C$ which tends to infinity at the rate of iterated exponentiation. This set must lie in the Julia set of $\gamma e^{z}+\delta e^{-z}$, and so the Julia set itself has positive measure, establishing Theorem 1.1.

Why is the last sentence of this proof true? One can prove by elementary arguments that for $f(z)=\gamma e^{z}+\delta e^{-z}$ or $\lambda e^{z}, f^{k}(w) \rightarrow \infty$ always implies $w$ is in the Julia set of $f$. Rather than proving this, we show in the Appendix that for a general class of entire functions (those of finite type), points which tend to infinity very rapidly (say at the rate of iterated exponentiation) lie in the Julia set. This is enough for the proof above. 


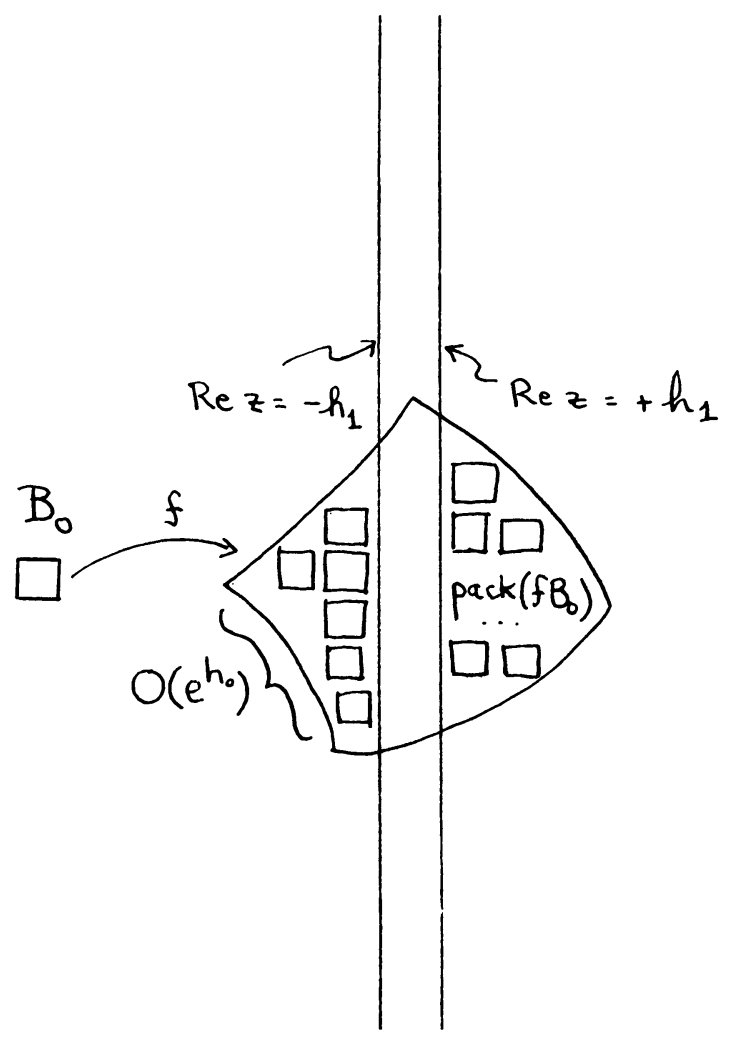

FIGURE 4.1. Dynamics of $\gamma e^{z}+\delta e^{-z}$

5. Behavior of $\lambda e^{z}$ : Dimension $=2$. Our study of $f(z)=\lambda e^{z}$ is rather similar to the discussion of $\sin (\alpha z+\beta)$ in $\S 4$. Only two properties of $f$ are essential: first, that $f(z)$ is rapidly expanding in the right half-plane; and second, that about half of the points in the right half-plane remain there after applying $f$.

To make this precise, let $I=\left[\theta_{0}-\arg \lambda, \theta_{1}-\arg \lambda\right]$ be an interval of angles; then $\arg f(x+i y)$ is in $\left[\theta_{0}, \theta_{1}\right]$ whenever $y \in I(\bmod 2 \pi)$. Set $\left[\theta_{0}, \theta_{1}\right]=[-\pi / 4, \pi / 4]$, and consider the region $S=\{z: \operatorname{Im}(z) \in I(\bmod 2 \pi)\}$. For $z$ in $S$, we have the estimate $\operatorname{Re} f(z)>O\left(e^{\operatorname{Re} z}\right)$. Thus as long as the iterates of a point remain in $S$, the point moves to the right at the rate of iterated exponentiation. We will show the set of points with this behavior always has dimension two (and by the Appendix, such points must lie in the Julia set).

It is useful to start out far enough to the right to assure that $f$ is very expanding; so choose $x_{0}$ such that $\left|f^{\prime}(z)\right| \gg 1$ when $\operatorname{Re}(z)>x_{0}$, and set $T=\{z \in S: \operatorname{Re}(z)>$ $\left.x_{0}\right\}$. As in the study of $\sin (\alpha z+\beta)$, we define a box to be a square region in $T$ of side length $r$, where $r$ is chosen small enough to insure that $f$ is injective on $B$ and that the image $f(B)$ is itself very nearly square-shaped. This is possible by bounded nonlinearity $\left(f^{\prime \prime} / f^{\prime}=1\right)$.

ProOF OF THEOREM 1.2 (The Julia set of $\lambda e^{z}$ has dimension 2). The proof parallels that for $\sin (\alpha z+\beta)$. Let $B$ be a box in $T$. Then the set $f(B) \cap T$ can itself be packed with disjoint boxes whose total density in $f(B)$ is near to $1 / 4$. This 


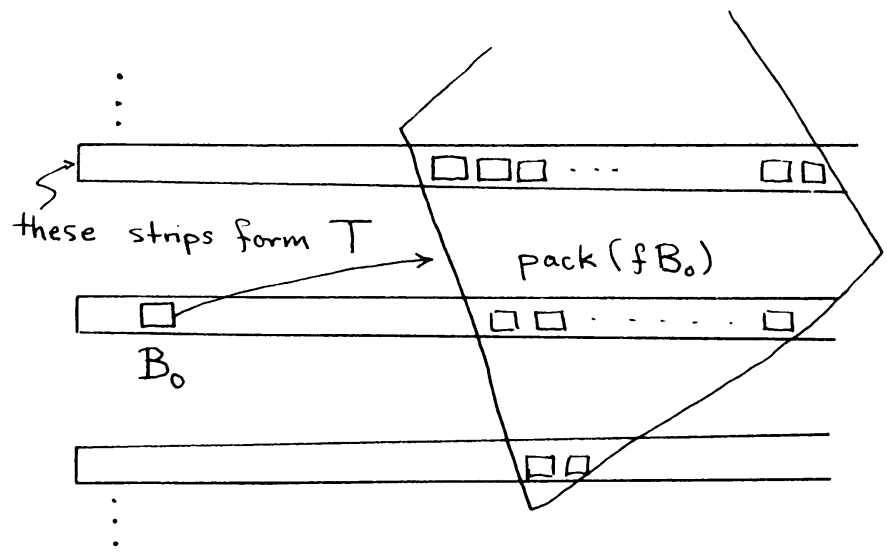

FigURE 5.1. Dynamics of $\lambda e^{z}$

follows from the fact that $f(B)$ is a very nearly square region much larger than the width of the strips forming $T$. As before we will refer to the boxes in such a packing as $\operatorname{pack}(f(B))$.

Let $E_{0}=\left\{B_{0}\right\}$ and define inductively

$$
E_{k}=\left\{G: G \subset F \in E_{k-1} \text {, and } f^{k}(G) \in \operatorname{pack}\left(F^{k} F\right)\right\} .
$$

$E_{k}$ consists of preimages of the boxes packing $f^{k}(F) \cap T$ for each $F$ in $E_{k-1}$ (see Figure 5.1). Then $E=\bigcap \bar{E}_{k}$ consists of points for which $f^{k}(z) \in T$ for all $k$.

Since density ( $\operatorname{pack}\left(f^{k} F\right), f^{k} F$ ) is nearly $1 / 4$, we conclude by the distortion lemma that density $\left(\bar{E}_{k+1}, F\right) \geq \Delta$ for some $\Delta$ independent of $k$. Using the fact that $\operatorname{Re} f(z)>O\left(e^{\operatorname{Re} z}\right)$ for $z \in T$, and $f^{\prime}(z)=f(z)$, one easily checks that for $z \in F \in E_{k}$,

$$
\operatorname{Re}\left(f^{k}(z)\right)>O\left(g^{k}(1)\right) \text { and diameter }(F)<d_{k}=O\left(1 / g^{k}(1)\right),
$$

where $g(x)=e^{x}$. Since $d_{k}$ tends to zero much faster than $\Delta^{k}$, Proposition 2.2 applies to prove the Hausdorff dimension of $E=\bigcap E_{k}$ is two. By the above, the iterates $\left\langle f^{k}(z)\right\rangle$ tend to infinity at the rate of iterated exponentiation for any $z$ in $E$; in particular, $E$ lies in the Julia set of $f$ (see the Appendix), establishing Theorem 1.2.

REMARK. The method of Proposition 2.2 shows in fact that the Julia set of $\lambda e^{z}$ has infinite Hausdorff measure for the gauge function

$$
h(r)=r^{2} \log \log \cdots \log (1 / r)
$$

for any number of iterations of the logarithm.

6. Expanding entire functions. To begin the proof of Theorem 1.3, we first develop general results concerning the expansion and distortion properties of certain entire functions.

DEFINITIONS. Let $f(z)$ be an entire function. A point $w \in \mathbb{C}$ is a singular value of $f$ if there is no neighborhood $U$ of $w$ such that $f: f^{-1}(U) \rightarrow U$ is a covering map. The post-singular set $P$ is the union of the singular values and their forward orbits; i.e.

$$
P=\left\{f^{n}(w): n \geq 0, \text { and } w \text { is a singular value of } f\right\} .
$$


We say $f$ is expanding if $\bar{P}$ is compact and $\bar{P} \cap J$ is empty (where $J$ is the Julia set of $f$ ). The terminology is justified by

PROPOSITION 6.1. If $f(z)$ is an expanding entire function, then $\left|f^{n^{\prime}}(z)\right| \rightarrow \infty$ for all $z$ in $J$.

PROOF. The proof uses the Poincaré metric as in Douady's proof of the corresponding result for rational functions $[D]$. Let $V$ denote the unbounded component of the complement of $\bar{P}$. We claim $J$ is contained in $V$; indeed, on any bounded component the boundary values of $f^{n}$ lie in the compact set $\bar{P}$ and by the maximum principle the iterates form a normal family.

It is easy to see that $U=f^{-1} V$ is contained in $V$ and $f: U \rightarrow V$ is a covering transformation. Except for the case of $f$ conformally conjugate to $f(z)=z^{n}$ (for which the proposition is true by inspection), $U$ is a proper subset of $V$. For if $U=V$ then $f$ is a polynomial (consider the preimage of a neighborhood of infinity) and the claim follows easily.

Let $\lambda(z)|d z|$ denote the Poincaré metric on $V$; then the Schwarz lemma shows that for $z$ in $U$ (in particular for $z$ in $J$ ),

$$
\left\|f^{\prime}(z)\right\|>1
$$

where $\|\cdot\|$ denotes the norm of the derivative of $f$ at $z$ in the $\lambda$ metric. To complete the proof we combine this fact with the observation that $\lambda(z) \rightarrow 0$ as $z \rightarrow \infty$.

The ordinary absolute value of the derivative of $f$ is related to the lambda norm by

$$
\left|f^{n \prime}(z)\right|=\left\|f^{n \prime}(z)\right\| \lambda(z) / \lambda\left(f^{n}(z)\right) .
$$

Fixing $z$ now, we distinguish two cases. If the iterates $f^{n}(z)$ return infinitely often to some fixed compact subset $K$ of $J$, then the derivative of $f^{n}$ in the lambda metric tends to infinity since $f$ expands by a definite amount on $K$. But $\lambda\left(f^{n}(z)\right)$ is bounded above (since $\lambda$ tends to zero at infinity) so $\left|f^{n \prime}(z)\right| \rightarrow \infty$ as claimed.

On the other hand, if $f^{n}(z)$ tends to infinity then $\lambda\left(f^{n}(z)\right) \rightarrow 0$ and since the $\lambda$-norm of $f^{n \prime}(z)$ is always $>1$ we have the same conclusion.

Using the more precise fact that $\lambda(z)$ is $O(1 /(|z| \log |z|))$ as $z$ tends to infinity (since $P$ is bounded), the same reasoning yields the following

COROLlaRY 6.2. If $f$ is expanding, then for all $z$ in $J$,

$$
\left|f^{n \prime}(z)\right| \geq C\left|f^{n}(z)\right| \log \left|f^{n}(z)\right|
$$

for some constant $C$ (depending on $z$ ).

The next result shows that a Julia set of an expanding entire function is quasiself-similar in the sense that all of its microscopic features are reflected with bounded distortion in its macroscopic features. This property is discussed for expanding rational functions by Sullivan $[\mathbf{S}]$. The implications for entire functions are not quite as strong as for rational functions because the Julia set is not compact. For example, the Julia set of an expanding rational function always has measure zero-this is a microscopic reflection of the macroscopic fact that $J$ is nowhere dense. But the corresponding result is not true for expanding entire functions-as we have seen, the Julia set of $f(z)=\sin (\alpha z+\beta)$ always has positive measure, even when $f$ is expanding. 
We will use the Koebe distortion theorem for univalent functions to control the shape of the Julia set of $f(z)$ when $f$ is expanding. The Koebe theorem is related to the distortion lemma introduced in $\S 3$, but is more generally applicable since it only requires the hypothesis of univalence. Indeed some of the preceding proofs could have relied on the Koebe theorem instead; however the arguments would become somewhat more cumbersome.

PROPOSITION 6.3 (KOEBE THEOREM). Let $B(r)$ denote the ball of radius $r$ centered at the origin, and let $g: B(r) \rightarrow \mathbb{C}$ be a univalent map. Then for any $s<r$, the restriction of $g$ to $B(s)$ has distortion bounded by a constant $K(s, r)$ tending to 1 as $s / r \rightarrow 0$.

Proof. See for example Ahlfors $[\mathbf{A}]$.

PROPOSITION 6.4. Let $f(z)$ be an expanding entire function. Then there is a countable family of balls $\left\{B_{\alpha}\right\}$ whose diameters tend to infinity, with the following property: every $z$ in the Julia set has a sequence of neighborhoods $U_{n}$ whose diameters tend to zero, such that $f^{n}$ maps $U_{n}$ onto a member of the family $\left\{B_{\alpha}\right\}$ with distortion bounded by a constant $L$ independent of $z$ and $f$.

PROOF. For $\left\{B_{\alpha}\right\}$ we take a locally finite covering of $J$ by balls with centers in $J$, each of the form $B(z, \operatorname{dist}(z, \bar{P}) / 2)$. Since $\bar{P}$ is compact, the diameter of balls in this family tends to infinity.

Now fix $z$ in $J$. For each $n$ let $B_{n}$ be a member of the family $\left\{B_{\alpha}\right\}$ containing $f^{n}(z)$. The critical values of $f^{n}$ are contained in $P$, so $f^{n}$ admits a univalent inverse on the ball with the same center as $B_{n}$ but twice the radius. Let $f^{-n}$ denote the branch of the inverse carrying $f^{n}(z)$ back to $z$, and let $U_{n}=f^{-n}\left(B_{n}\right)$. By the Koebe theorem this map has distortion bounded by a universal constant. It remains only to verify that the diameters of the $U_{n}$ 's tend to zero.

By bounded distortion,

$$
\operatorname{diam}\left(U_{n}\right)=O\left(\operatorname{diam}\left(B_{n}\right) /\left|f^{n \prime}(z)\right|\right) .
$$

By Proposition $6.1,\left|f^{n \prime}(z)\right| \rightarrow \infty$, so we need only worry about the $n$ such that $\operatorname{diam}\left(B_{n}\right)$ is large. But for such $n, \operatorname{diam}\left(B_{n}\right)$ is comparable to $\left|f^{n}(z)\right|$, and applying Proposition 6.2 we have $\operatorname{diam}\left(U_{n}\right)=O\left(1 / \log \left|f^{n}(z)\right|\right)$, so the diameters tend to zero for these $n$ as well.

7. Behavior of $\lambda e^{z}:$ Area $=0$. Using the results of the preceding section, we can now easily show that if the Julia set of an expanding function is "thin at infinity" then its area is zero. We begin by establishing some properties of $\lambda e^{z}$ which make it clear that this result includes Theorem 1.3 as a special case.

Proposition 7.1. If $\lambda e^{z}$ has an attracting periodic cycle then it is expanding.

In fact the converse is true (but we do not use this).

PROOF. Assume $f$ has an attracting cycle. Note that $f$ has only one singular value (namely 0 ) and any attracting cycle must attract this value (by the usual Schwarz lemma argument-otherwise $f$ is a local isometry in the Poincaré metric on the immediate basic of attraction). Since some neighborhood of the attracting cycle does not meet the Julia set, we conclude that $\bar{P} \cap J$ is empty and $f$ is expanding. 
Theorem 1.3 clearly depends on some property besides expansion, since the Julia set of an expanding entire function can have positive area. To isolate this property, we say a subset $E$ of $\mathbf{C}$ is thin at infinity if its density is bounded away from 1 in all sufficiently large balls: there exists an $R$ and an $\varepsilon$ such that for all $z$, $\operatorname{density}(E, B(z, R))<1-\varepsilon$.

PROPOSITION 7.2. When $\lambda e^{z}$ is expanding, its Julia set is thin at infinity.

Proof. For $\lambda e^{z}$ expanding a neighborhood $U$ of 0 is in the complement of $J$ (since 0 is a singular value); the preimage of $U$ contains some left half-plane $H$, and the preimage of $H$ consists of periodic horizontal strips $S$. Any sufficiently large ball must either lie in $H$ or meet a definite amount of $S$.

A set $E$ in $\mathbf{C}$ is said to be totally invariant under $f$ if $f^{-1}(E)=E$. Since the Julia set is totally invariant, Theorem 1.3 is a special case of

PROPOSITION 7.3. Let $f(z)$ be an expanding entire function and suppose $E$ is a measurable totally invariant subset of the Julia set of $f$. Then if $E$ is thin at infinity, its area is zero.

This general form will prove useful in studying the measurable dynamics of $\sin (\alpha z+\beta)$.

PROOF. The proof parallels Sullivan's argument for rational functions $[\mathbf{S}]$. For $f(z)$ expanding, we may choose a family of balls $\left\{B_{\alpha}\right\}$ as in the statement of Proposition 6.4. The diameters of these balls tend to infinity and $E$ is thin at infinity, so there is a uniform upper bound to the density of $E$ in these balls. Since $E$ is totally invariant, we may apply Proposition 6.4 to conclude that every point in $E$ has arbitrarily small neighborhoods in which the density of $E$ is less than one by a definite amount. Furthermore these neighborhoods have bounded geometry-they are images of round balls under maps of uniformly bounded distortion. Therefore $E$ has no points of Lebesgue density and consequently its area is zero.

8. Measurable dynamics of $\sin (\alpha z+\beta)$. The results in $\S 3$ indicate that many points in the Julia set of $f(z)=\sin (\alpha z+\beta)$ tend towards infinity at a definite rate. In this section we construct a function which measures this tendency and establishes a measurable semiconjugacy between the action of $f$ on its Julia set and the action of $g(x)=e^{x} / e$ on $[1, \infty]$. It will turn out that the push-forward of area measure on $J$ gives positive measure to any subinterval of $(1, \infty)$; since the action of $g$ is properly discontinuous on this open set, it follows immediately that $f$ is not ergodic.

As usual, it will be more convenient to put the function $f$ in the form $f(z)=$ $\gamma e^{z}+\delta e^{-z}$.

Define, for all $z$ in $\mathbf{C}$,

$$
\phi(z)=\inf \left\{x \geq 1: \lim _{n \rightarrow \infty} \frac{\left|f^{n}(z)\right|}{g^{n}(x)}=0\right\} .
$$

This is a semiconjugacy: $\phi(f(z))=g(\phi(z))$, as is easily verified. A computation establishes

$$
\phi(z) \leq|\operatorname{Re}(z)|+\varepsilon(|\operatorname{Re} z|)
$$

where $\varepsilon \rightarrow 0$ as $|\operatorname{Re} z| \rightarrow \infty$. (In particular, $\phi(z)$ is always finite.) Here is a sort of converse. 
Proposition 8.1. For any fixed $\varepsilon$, let $E=\{z:|\phi(z)-| \operatorname{Re} z||<\varepsilon\}$. Then density $(E, B(z, 1)) \rightarrow 1$ as $|\operatorname{Re} z| \rightarrow \infty$. In other words, when $|\operatorname{Re} z|$ is large, $\phi(z)$ is very probably quite close to $|\operatorname{Re} z|$.

ProOF. Let $x=|\operatorname{Re} z|$. A calculation shows we can choose a sequence $\alpha_{1}>$ $\alpha_{2}>\alpha_{3}>\cdots$ such that $\varepsilon=\alpha_{1}$ and

$$
\sum_{k=0}^{\infty} \frac{g^{k+1}\left(x-\alpha_{k+1}\right)}{g^{k+1}\left(x-\alpha_{k}\right)} \rightarrow 0 \quad \text { as } x \rightarrow \infty .
$$

Applying Proposition 3.2 with $h_{k}=g^{k}\left(x-\alpha_{k}\right)$, we conclude that in a box centered at $z$, the density of

$$
\left\{w:\left|\operatorname{Re} f^{k}(w)\right| \geq g^{k}\left(|\operatorname{Re} z|-\alpha_{k}\right) \text { for all } k\right\}
$$

tends to 1 as $|\operatorname{Re} z| \rightarrow \infty$. But clearly for any such $w, \phi(w) \geq|\operatorname{Re} z|-\varepsilon$. Combining this with the preceding upper bound on $\phi(z)$ yields the proposition.

COROLLARY 8.2. $\phi^{-1}(I)$ has positive area for any interval I of positive length contained in $[1, \infty)$.

ProOF. By the proposition above, $\{z: \phi(z) \in[x-1, x+1]\}$ has positive area for $x$ large enough, since it contains all the $z$ such that $|\operatorname{Re} z|$ is near $x$. But for any interval $I, g^{n}(I)$ contains an interval of the form above once $n$ is large enough. Since $f$ is absolutely continuous with respect to area measure and $\phi$ is a semiconjugacy, we conclude that $\phi^{-1}(I)$ itself has positive area.

PROOF OF THEOREM 1.4 ( $f$ is not ergodic on its Julia set). First, notice that any $z$ such that $\phi(z)>1$ tends to infinity at the rate of iterated exponentiation and consequently (by the Appendix) lies in the Julia set. Let $I$ and $J$ be any two intervals in $(1, \infty)$ such that their grand orbits under $g$ are disjoint (such $I$ and $J$ clearly exist since $g$ has a fundamental domain). Then the grand orbits under $f$ of $\phi^{-1}(I)$ and $\phi^{-1}(J)$ are also disjoint, because $\phi$ is a semiconjugacy. By the preceding corollary, these are subsets of $J$ of positive measure; so $f$ is not ergodic.

When $f$ is expanding the action of $g$ on $(1, \infty)$ seems to entirely capture the measurable dynamics of $f$. One aspect of this is the following:

PROPOSITION 8.3. If $f(z)$ is expanding, then the area of $J \cap \phi^{-1}(1)$ is zero.

Thus in the expanding case almost every point in the Julia set tends to infinity at the rate of iterated exponentiation.

Proof. $J \cap \phi^{-1}(1)$ is totally invariant, and by Proposition 8.1 it is thin at infinity; so by Proposition 7.3 it has measure zero.

We conjecture that when $f$ is expanding, the semiconjugacy $\phi$ gives exactly the measurable small-orbit quotient for the action of $f$ on $J$.

Appendix: Points zipping towards infinity are in the Julia set. An entire function is said to be of finite type if it has finitely many singular values (see $\S 6)$.

Proposition A.1. Let $f(z)$ be an entire function of finite type and let $w$ be a point not lying in the Julia set of $f$. Then there exist a constant $P>1$, such that $\log \left|f^{k}(w)\right| \leq O\left(P^{k}\right)$.

PROOF. By Goldberg and Keen [GK], functions of finite type have no wandering domains; so the forward orbit of $w$ eventually lands in a component $U$ of the 
complement of the Julia set which is fixed by a power of $f$. For simplicity, assume this power is one, and assume $w$ was in $U$ to start with. Let $d(z, w)$ denote the Poincaré metric on $U$. By the Schwarz lemma, $f: U \rightarrow U$ is distance nonincreasing for this metric. Since inclusions are contracting, $d(z, w) \geq D(z, w)$ where $D$ denotes the Poincaré metric on $\mathbb{C}-\{a, b\}$ for $\{a, b\}$ two points chosen to lie outside of $U$.

Using estimates for the Poincaré metric on a triply punctured sphere (see Ahlfors $[\mathbf{A}])$, we find that for $z, w$ large,

$$
D(z, w) \geq O(|\log \log | z|-\log \log | w||) .
$$

Therefore,

$$
\begin{aligned}
& C|\log \log | f^{k+1}(w)|-\log \log | f^{k}(w)|| \\
& \quad \leq D\left(f^{k+1}(w), f^{k}(w)\right) \leq d\left(f^{k+1}(w), f^{k}(w)\right) \leq d(f(w), w)
\end{aligned}
$$

for some positive constant $C$. Since the quantity on the right is independent of $k$, an easy computation now yields the proposition.

REMARKS. (1) The rate of approach $P^{k}$ is realized in the basin of attraction of infinity when $f$ is a polynomial of degree $P$.

(2) Since $\lambda e^{2}, \sin (\alpha z+\beta)$ and $\cos (\sqrt{\alpha z+\beta})$ are of finite type, any point whose forward orbit under one of these functions tends to infinity at the rate of iterated exponentiation must lie in the Julia set. (If $g(x)=e^{x}$, then for $k$ large enough,

$$
\log g^{k}(1)=g^{k-1}(1)>C P^{k}
$$

for any given $C$ and $P$.) This fact was used in the proofs of Theorems 1.1, 1.2 and 1.4 .

(3) The example $f(z)=e^{-z}+z+1$ shows that an entire function can have an open set which is attracted to infinity; therefore $f^{k}(z) \rightarrow \infty$ does not always assure $z$ is in the Julia set. This example, which is due to Fatou [F], was pointed out by Lisa Goldberg. Notice Fatou's example is not of finite type. Is there a finite-type map with a basin at infinity?

ADDED IN PROOF. A negative answer to this question has been announced by Eremenko and Ljubich $[\mathbf{E L}]$.

\section{REFERENCES}

[A] L. Ahlfors, Conformal invariants, McGraw-Hill, New York, 1973.

[B1] I. N. Baker, The domains of normality of an entire function, Ann. Acad. Fenn. Ser. AI 1 (1975), 277-283.

[B2] _ Some entire functions with multiply-connected wandering domains, Ergodic Theory Dynamical Systems 5 (1985), 163-169.

[BR] I. N. Baker and P. J. Rippon, Iteration of exponential functions, Ann. Acad. Fenn. Ser. AI 9 (1984), 49-77.

[DK] R. Devaney and M. Krych, Dynamics of $\operatorname{Exp}(z)$, Ergodic Theory Dynamical Systems 4 (1984), 35-52.

[D] A. Douady, Systèmes dynamiques holomorphes, Asterisque 105 (1983), 39-63; Ergodic Theory Dynamical Systems 4 (1984), 35-52.

[EL] A. È. Eremenko and M. Yu. Lyubich, Iterates of entire functions, Soviet Math. Dokl. 30 (1984), 592-594.

[F] M. P. Fatou, Sur l'iteration des fonctions transcendentes entières, Acta Math. 47 (1926), 337-370.

[G] F. Gehring, Injectivity of local quasi-isometries, Comm. Math. Helv. 57 (1982), $202-220$. 
[GGS] E. Ghys, L. Goldberg and D. Sullivan, On the measurable dynamics of $z \rightarrow e^{z}$, Ergodic Theory Dynamical Systems 5 (1985), 329-335.

[GK] L. Goldberg and L. Keen, $A$ finiteness theorem for a dynamical class of entire functions, Ergodic Theory Dynamical Systems 6 (1986), 183-192.

[J] F. John, On quasi-isometries. I, Comm. Pure Appl. Math. 21 (1968), 77-110.

[KS] J. P. Kahane and R. Salem, Ensembles parfaits et séries trigonométriques, Paris, Hermann, 1963, p. 27.

[M] M. Misiurewicz, On iterates of $e^{z}$, Ergodic Theory Dynamical Systems 1 (1981), 103-106.

[S] D. Sullivan, Conformal dynamical systems, Geometric Dynamics, Lecture Notes in Math., vol. 1007, Springer-Verlag, 1983, pp. 725-752.

Mathematical Sciences Research Institute, 1000 Centennial Drive, BerkeLEY, CALIFORNIA 94720

Current address: School of Mathematics, Institute for Advanced Study, Princeton, New Jersey 08540 